\title{
A Parametric Analysis of Finite Phased Arrays of Printed Dipoles on Large Circular Cylinders and Comparisons with the Planar Case
}

\author{
B. Güner, V. B. Ertürk* and O. Bakar \\ Electrical \& Electronics Eng. Dept., Bilkent Univ., TR-06800 Ankara, Turkey. \\ E-mail; vakur@ee.bilkent.edu.tr, Tel: ++90312 2903154
}

\section{INTRODUCTION}

Although the primary concern in the design of antennas is to be able to send and receive information in a fast and accurate way, there are other important practical constraints to be met. Many military and commercial applications require the use of antennas that conform to the main body. For example, when an antenna is mounted on the surface of a ship, aeroplane or a missile, conformality to the surface is generally aimed due to the reasons like aerodynamic constraints, reduced radar cross section (RCS), space limitations or aesthetic concerns. However, the design and analysis tools available for the conformal arrays do not provide enough efficiency and accuracy to be practically useful, especially for electrically large, cylindrically shaped coated host bodies.

Recently, a hybrid MoM/Green's function technique in the spatial domain is developed, which improves the efficiency and accuracy problems in the analysis of conformal arrays [1], [2]. In this paper, this technique is used to perform a full-wave analysis of large phased arrays of printed dipoles on electrically targe material coated circular cylinders of both axially and circumferentially oriented dipoles. The effects of several array and supporting structure parameters are observed. Results are also compared with the corresponding planar arrays. Consequently, a complete understanding on the performance of arrays of printed dipoles on large coated cylinders with respect to several array parameters (i.e. the dielectric constant, thickness of the coating material, the direction of the dipoles) as well as the effects of curvature (compared to planar arrays) are investigated.

\section{FORMULATION}

The geometry consists of finite, periodic arrays of $(2 \mathrm{~N}+1) \times(2 \mathrm{M}+1)$ identical $z$-directed and $\Phi$-directed printed dipoles. The dipoles are mounted on the air-dielectric interface of an infinitely long, cylindrically shaped grounded dielectric substrate with an inner radius $a$, outer radius $d$, thickness $t_{h}=d-a$ and relative permittivity $\varepsilon_{r}>1$. Furthermore, the dipoles are assumed to be center-fed with an ideal delta gap generator, and each dipole has a generator terminating impedance $Z_{\mathrm{T}}$. The hybrid MoM/Green's function solution for this problem follows the following procedure:

By forming an electrical field integral equation (EFIE) such that the total electrical field in the direction of the orientation of the dipole must vanish on the dipole surfaces, and using Galerkin's MoM solution, the following matrix equation is obtained [3]:

$$
\left([\mathbf{Z}]+\left[\mathbf{Z}_{\mathbf{T}}\right]\right) \cdot \mathbf{I}=\mathbf{V} .
$$

In (1), $\mathbf{V}$ is the voltage vector which is related to the excitation of the dipoles. Using a single expansion mode for each dipole, the voltage vector element for the $\mathrm{pq}^{\text {th }}$ dipole becomes 


$$
V_{p q}=e^{-j k_{0} \sin \theta_{i} d \cos \left(\phi_{i}-p \Delta \phi\right)} e^{-j k_{0} \cos \theta_{i} q d_{z}}
$$

where $\left(\theta_{i}, \phi_{i}\right)$ being the scan direction of the beam. $I=\left[1_{n m}\right]$ is the unknown vector of expansion coefficients (current vector) whose values are going to be found via MoM procedure, $\left[\mathbf{Z}_{\mathrm{r}}\right]$ is the diagonal generator terminating impedance matrix, and finally $[\mathbf{Z}]$ is the impedance matrix of the array elements whose entries are given by

$$
Z_{n m, p q}=\iint_{s_{\mathrm{p}}} d s_{p q} \iint_{s_{\infty m}} d s_{\mathrm{nm}}^{\prime} \mathbf{f}_{\mathrm{pq}}\left(\mathbf{r}_{\mathrm{pq}}\right) \mathrm{G}_{\mathrm{uu}}\left(\mathbf{r}_{\mathrm{pq}} / \mathbf{r}_{\mathrm{nm}}^{\prime}\right) \mathbf{f}_{\mathrm{nm}}\left(\mathbf{r}_{\mathrm{nm}}^{\prime}\right) .
$$

In (3) $r_{p q}$ and $r_{n m}^{\prime}$ are the position vectors of the $p q^{\text {th }}$ and $n m^{\text {th }}$ dipoles, $G_{u u}\left(r_{p q} / r_{n m}^{\prime}\right)$ is the appropriate dyadic Green's function component and $f_{n m}\left(r_{n m}^{\prime}\right)$ and $f_{p q}\left(r_{p q}\right)$ are the piece-wise sinusoidal basis and testing functions, respectively.

As the array size becomes larger, the importance of the fast and accurate calculation of the impedance matrix increases. This is achieved via efficient and accurate calculation of the Green's function $G\left(r / r^{\prime}\right)$ component for arbitrary source and observation locations. Therefore, three different spatial domain representations are used to find the Green's function in the most efficient and accurate way, all of which are valid in different but overlapping regions of space.

The first one is a steepest descent path (SDP) representation [4], which is valid at the off-paraxial region. This representation is based on the efficient numerical evaluation of a circumferentially propagating series representation of the appropriate Green's function along the SDP on which the integrand decays most rapidly. The second representation is the paraxial spatial domain representation [5], which is valid along the paraxial region. In this representation, we approximate the Green's function by a Fourier Series (FS) using the periodicity of the circumferentially propagating series representation of the appropriate Green's function in one of its variables. The coefficients of the FS expansion can be found by a simple numerical integration algorithm. The algorithm used determines the accuracy and efficiency of this method. Including only the two leading terms of the FS expansion gives enough accuracy for most cases. Both SDP and FS representations looses their accuracy when the separation between the source and observation points is small. Since this work is focusing on electrically large cylinders, for small separations cylinder surface can be considered locally flat [6]. Hence, planar approximations are used for self-term evaluations.

Several performance metrics [3], [7] for both axially and circumferentially oriented finite printed dipoles on coated cylinders, the effects of array and host body parameters on their behavior, as well as the effects of he curvature and array element orientation are investigated after solving the matrix equation given by (1).

\section{NUMERICAL RESULTS}

Figure 1.a and Figure 1.b illustrate the magnitude of the reflection coefficient versus scan angle of $11 \times 11 \Phi$ - and $z$-directed dipole arrays on a dielectric coated circular cylinder with $a=3 \lambda_{0}, t_{h}=$ $0.06 \lambda_{0}, \varepsilon_{r}=3.25$ as well as the same result for a planar arsay along the E- and H-planes, respectively. The dipole dimensions are $\left(0.39 \lambda_{0}, 0.01 \lambda_{0}\right)$ for the length and width of each dipole, respectively and the dipoles are uniformly spaced from their neighbors by distances of $0.5 \lambda_{0}$ in $z$ and $\phi$-directions ( $x$ - and $y$-for planar case), respectively. It is seen that the array of axially oriented dipoles on the cylinder shows a scan blindness along the E-plane (the magnitude of the reflection coefficient exceeds unity) at approximately $48^{\circ}$. However, the scan blindness 
phenomenon is observed for neither the array of circumferentially oriented dipoles on the cylinder nor the planar array. Along the H-plane, none of the arrays shows a scan blindness. Since scan blindness is related with the surface wave fields excited within the substrate, the curvature effects as well as the orientation of the array elements will change the behavior of these fields.

In Figure 2, the change in the magnitudes of the currents on the dipoles for the middle row (E-plane) of a $11 \times 11 \phi$-directed array is shown when $a$ is varied between $2.5 \lambda_{0}$ and $5 \lambda_{0}$, and they are compared with the corresponding planar array with all other parameters being the same as above. Although the currents of the dipoles are close to each other for cylindrical arrays with different inner radii, it can still be seen that as the cylinder radius increases the current levels become closer to the planar array results which is the limiting case of the cylindrical array when the radius goes to infinity.

Figure 3 shows the normalized E-plane far-field pattern of $11 \times 11 \Phi$ - and $z$-directed dipole arrays on a dielectric coated circular cylinder, and an $11 \times 11$ planar array for a $45^{\circ}$ scan angle with the same parameters used as in Figures 1.a and 1.b. It is seen that although the pattern for the cylindrical and planar cases are close for the main lobe, away from the main lobe a significant difference occurs due to the curvature of the cylinder. The sidelobe levels for the cylindrical arrays are higher since some of the element patterns have their peaks other than the intended main beam direction since no special beam-forming technique is used.

\section{CONCLUSIONS}

A parametric study of finite phased arrays of printed dipoles on electrically large coated cylinders is performed using a spatial domain hybrid MoM/Green's function technique. Dipoles' currents and several performance metrics like active reflection coefficient and input impedances of the dipoles are calculated and compared with their planar counterparts. The effects of the curvature, dipole orientation and changes in the array and host body parameters are observed and discussed. Several more numerical examples will be presented during the presentation.

\section{REFERENCES}

[1] V. B. Ertürk, R. G. Rojas and K. W. Lee, "Analysis of finite arrays of axially directed printed dipoles on electrically large circular cylinders", accepted to IEEE Trans. Antennas and Propagat.

[2] B. Güner and V. B. Ertìrk, "Analysis of finite arrays of circumferentially oriented printed dipoles on electrically large cylinders", 2003 IEEE AP-S Int. Symp. And USNC/CNC/URSI National Radio Science Meeting, Columbus, OH, June 22-27 2003.

[3] D. M. Pozar, "Analysis of finite phased arrays of printed dipoles", IEEE Trans. Antennas and Propagat., vol.33, no.10, pp. 1045-1053, Oct. 1985.

[4] V. B. Ertilrk and R. G. Rojas, "Efficient computation of surface fields excited on a dielectric coated circular cylinder", IEEE Trans. Antemnas and Propagat., vol.48, pp. 1507-1516, Oct. 2000.

[5] V. B. Erturk and R. G. Rojas, "Paraxial space-domain formulation for surface fields on dielectric coated circular cylinder", IEEE Trans. Antennas and Propagat., vol.50, pp. 1577-1587, Nov. 2002.

[6] S. Barkeshli, P. H. Pathak and M. Marin, "An asymptotic closed-form microstrip surface Green's function for the efficient moment method analysis of mutual coupling in microstrip antennas", IEEE Trans. Antennas and Propagat., vol.38, pp. 1374-1383, Sept. 1990.

[7] R. C. Hansen, Phased Array Antennas. New York, John Wiley \& Sons Inc., 1998.

[8] J. Ashkenazy, S. Shtrikman and D. Treves, "Electric surface current model for the analysis of microstrip antennas on cylindrical bodies", IEEE Trans. Antennas and Propagat., vol.33, pp. 295-300, March 1985. 

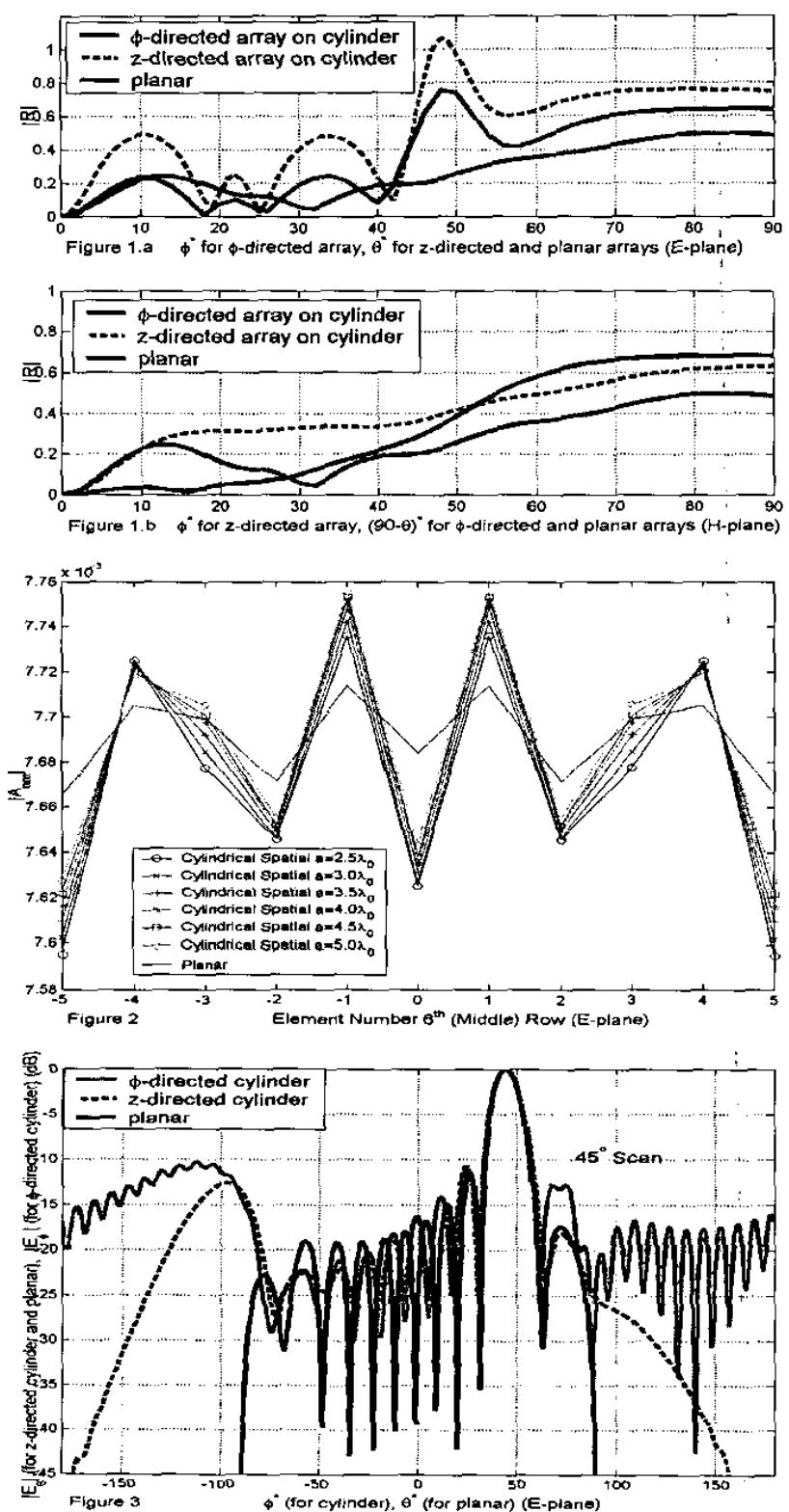